\title{
Regional differences in long-term cycles and seasonality of Puumala virus infections, Finland, 1995-2014
}

\author{
J. SANE ${ }^{1 *}$, J. OLLGREN ${ }^{1}$, P. MAKARY ${ }^{1}$, O. VAPALAHTI ${ }^{2,3,4}$, M. KUUSI $^{1}$ AND \\ O. LYYTIKÄINEN ${ }^{1}$ \\ ${ }^{1}$ Department of Infectious Diseases, Infectious Disease Control Unit, National Institute for Health and Welfare, \\ Helsinki, Finland \\ ${ }^{2}$ Department of Virology, Haartman Institute, University of Helsinki, Helsinki, Finland \\ ${ }^{3}$ Department of Veterinary Biosciences, University of Helsinki, Helsinki, Finland \\ ${ }^{4}$ HUSLAB, Department of Virology and Immunology, Hospital District of Helsinki and Uusimaa, Helsinki, \\ Finland
}

Received 10 November 2015; Final revision 9 March 2016; Accepted 31 March 2016;

first published online 26 April 2016

\section{SUMMARY}

Puumala hantavirus (PUUV) causes haemorrhagic fever with renal syndrome in humans, an endemic disease in Finland. We aimed to study recent trends in PUUV infections in Finland, to evaluate whether there are regional differences in seasonality and long-term cycles and whether the patterns have changed over time. We analysed serologically confirmed acute PUUV infections reported to the National Infectious Disease Register from 1 April 1995 to 31 March 2014. A total of 30942 cases of PUUV infections were identified during the study period. The average annual incidence was 31 cases/100 000 person-years with the highest in Eastern Finland and the lowest in Southwestern Finland. Throughout Finland there was not an increasing trend in incidence but changes in incidence, seasonality and long-term cycles differed regionally. Longterm cycles supported by high Bayesian posterior probabilities (73-100\%) differed between the south and the north, shifting from 3 to 4 years, respectively. Temporal changes in seasonality were most prominent in Southwestern Finland. The pattern of human PUUV infection epidemiology probably primarily reflects the spatio-temporal interaction between bank-vole population dynamics and climate.

Key words: Epidemiology, hantavirus, statistics, surveillance, zoonoses.

\section{INTRODUCTION}

Hantaviruses of the family Bunyaviridae, genus Hantavirus are predominantly rodent-borne pathogens that may cause haemorrhagic fever with renal syndrome (HFRS) in Eurasia [1]. Humans mainly become infected by inhaling aerolized rodent excreta

\footnotetext{
* Author for correspondence: Dr J. Sane, National Institute for Health and Welfare (THL), Mannerheimintie 166, FI-00271 Helsinki, Finland.

(Email: jussi.sane@thl.fi)
}

$[1,2]$. Five hantaviruses circulate among rodents in Europe, but the majority of human HFRS cases are caused by Puumala virus (PUUV) [1,3]. The bank vole (Myodes glareolus) is the reservoir for PUUV and is a widely spread vole species in Europe although absent in many areas around the Mediterranean [1]. HFRS is a reportable disease in many countries in the European Union. However, most cases are reported from Finland ( $\sim 70 \%$ of cases), Sweden and forest-rich regions of Belgium and Germany [4-8].

PUUV infections show pronounced seasonality in Finland with highest incidence rates in late summer 
and early winter [7, 9-11]. The bank vole undergoes 3to 4-year population cycles, seemingly driven by specialist predators, which coincide with human HFRS epidemics [1]. However, it has been unclear how seasonality and long-term cycles of these epidemics vary regionally and temporally.

The aim of this study was to report recent trends in PUUV infections in Finland and to evaluate in detail how different regions contribute to the general epidemiological pattern in Finland; whether there are regional differences in seasonality and long-term cycles and whether the patterns have changed over time.

\section{METHODS}

Since 1995, all Finnish clinical microbiology laboratories have reported serological tests that were positive for PUUV to the National Infectious Disease Register (NIDR). Each notification includes data on age, sex and, as well as date of diagnostic specimen, place of residence and treatment. Multiple notifications of persons with the same date of birth, sex and place of treatment received within a 12-month period were combined as one report; since 2004, this was done by a unique national identity number.

A case was defined as a person who had a positive serological test for PUUV infection which was reported to the NIDR from 1 April 1995 to 31 March 2014. We considered each 12-month period from April until the following March as a 'seasonal year', based on previous observation: the lowest number of cases occurred from March to April in each year. Data from the Population Information System for 1995-2014 were used as a denominator to calculate incidence rates. For regional analyses, we divided Finland into six regional states according to the division of Regional State Administrative Agencies (Fig. 1). This division follows the natural geographical division of Finland into Southern, Southwestern, Western \& Inland, Eastern, and Northern Finland as well as Lapland.

To estimate how different regions contribute to the general epidemiological pattern and to better understand the long-term cycles of PUUV infections in Finland, we constructed a first-order Poisson autoregression model using a Bayesian approach [12]. We estimated the parameters under a model-averaging perspective allowing for model uncertainty. We estimated the probability of different long-term cycles being in the model and Gibbs variable selection was performed [13]. We included cycles from 1.5 years to
4 years with increments of 0.5 years. Cycles with a $>50 \%$ inclusion probability were considered significant cycles. We used weakly informative priors. To control the correlation of errors, we made a predictive check on overdispersion.

We calculated the average monthly incidence/ 100000 person-years with $95 \%$ credible intervals in each regional states using the same model. To study changes in seasonality and incidence, we divided the study into three periods: first period (1 April 1995 to 31 March 2001), second period (1 April 2001 to 31 March 2007) and third period (1 April 2007 to 31 March 2014). A negative binomial regression model was used to estimate differences in incidence rate ratios between time periods. The analyses were performed with Stata v. 13 (Stata Corp., USA) and WinBUGS v. 1.4 .3 (http://www.mrc-bsu.cam.ac.uk/ software/bugs/). Figures were created by Excel (Microsoft Corp., USA).

\section{RESULTS}

\section{Descriptive data}

A total of 30942 cases of PUUV infection were identified during the study period (range by seasonal year 559-3779). Cases were mostly (61\%) males (median age 48, range 3-92 years). Most cases were notified from Eastern Finland $(n=9445,31 \%)$ and least cases from Southwestern Finland $(n=1534,5 \%)$. The average annual incidence was 31 cases $/ 100000$ personyears, with the highest in Eastern Finland and the lowest in Southwestern Finland (range by regional state $11 \cdot 1-80 \cdot 5$, range by seasonal year $10 \cdot 8-71 \cdot 2$ ).

\section{Changes in incidence over-time}

Overall, the model including the quadratic trend had the best fit (based on Akaike's Information Criterion) for describing the long-term trend in Finland. Compared to the first time period (reference), the average monthly incidence increased significantly over the three time periods in Southern and Eastern Finland. In Southwestern, and Western \& Inland Finland incidence increased from the first to second period but somewhat decreased or remained stable in the third period (Table 1). In Northern Finland and Lapland, incidence was higher during the second period compared to the first but not significantly different in the third period (Northern Finland) and in fact lower (Lapland) from the first time period. 


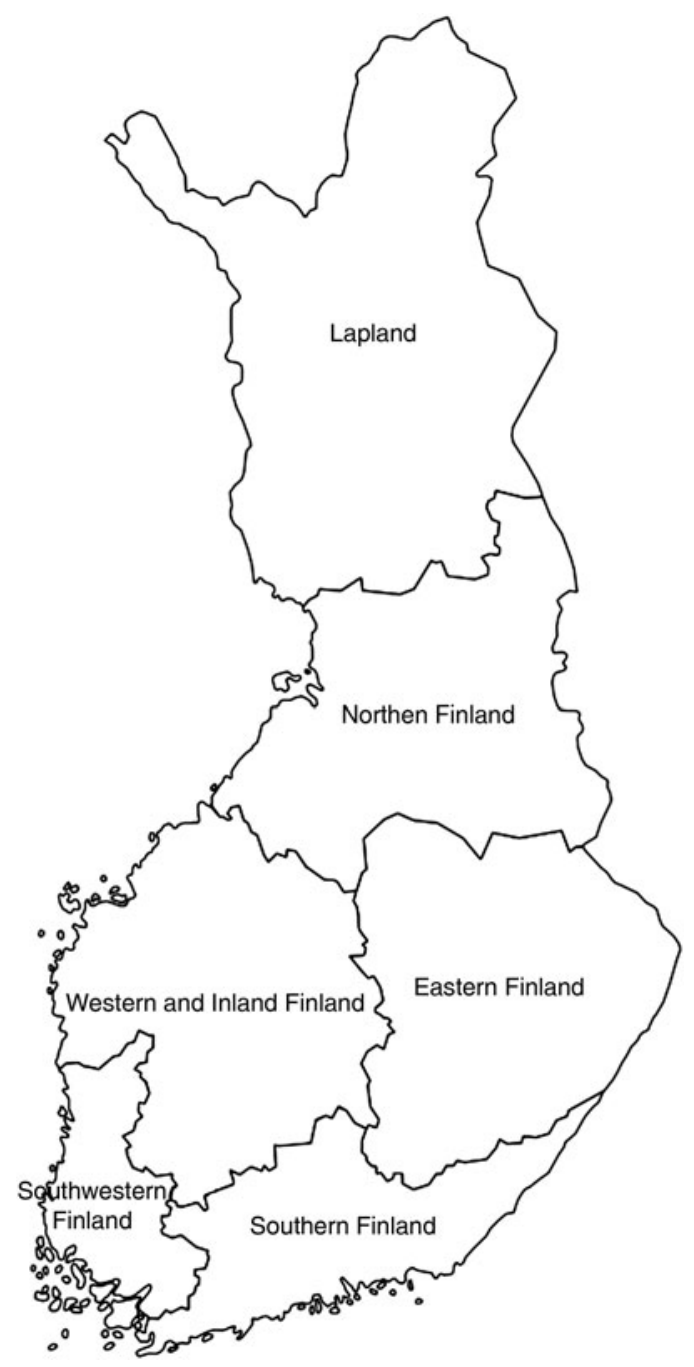

Fig. 1. Geographical division showing the regional states in Finland.

\section{Long-term cycles and seasonality}

The model fitted well with the actual observations in all the regional states: the model fit is shown in Supplementary Figure 1 for Southern Finland. Based on the posterior probabilities of different longterm cycles, five regional states had 3-year cycles with high posterior probability while a 4 -year cycle with $100 \%$ probability was observed in Lapland. The 3-year cycle probabilities for different regions were 73\% (Southern), 100\% (Southwestern), 86\% (Western \& Inland), $86 \%$ (Eastern) and 74\% (Northern). Other cycle probabilities in the model were negligible except for a $22 \%$ probability for a $3 \cdot 5$-year cycle in Northern Finland. The peak years differed between regions. Incidence peaked in 2008 in Southern, Southwestern, Western \& Inland, and Eastern
Finland while the peak was observed in 2007 in Northern Finland and Lapland.

The seasonality of incidences in Southern Finland was characterized by two peaks; the first in August and the second in early winter (Fig. 2). The timing of the second peak differed between the studied periods; in the first time period, a weak peak was observed in December but during the second and third periods the incidence peaked in November. In the third period, the average monthly incidence was highest in winter, not in August as in the other periods and incidence remained high until January.

In Southwestern Finland, a minor peak in October was observed in the first period, which was not evident in the second and third periods (Fig. 3). Incidence peaked in early winter during the third period (December) but later in winter during the second period (January); notably, similar winter peaks were not observed in the first period at all and overall, seasonality with two distinct peaks was most apparent in the third period. In Western \& Inland Finland, seasonality remained similar throughout the time periods with minor peaks in August followed by major peaks in early winter. The seasonality in Eastern Finland was distinctly different from Southern, and Western \& Inland Finland. Here the incidence peaked in early winter (December) with other seasonal peaks absent (data not shown).

In Northern Finland, there were some differences in the seasonal pattern between the time periods: the major peak in winter was observed earlier (November) in the second period than in the first and third periods (December). Furthermore, an additional increase in average incidence was observed in January during the second period after a significant decrease in December. In Lapland, the seasonal pattern was characterized by the early winter peak in November (Fig. 4). Incidence remained high in December in the first period. In the second period, incidence increased again in January after a drop in December, analogous to Northern Finland.

\section{DISCUSSION}

We report that PUUV infections are not increasing uniformly across Finland, but rather, changes in incidence, seasonality and long-term cycles differ regionally. Incidence of PUUV infection, which is reportedly the highest in the world, increased from the first to second period in all regions albeit subsequently decreased or remained stable in most regions. 
Table 1. Incidence rate ratios of Puumala virus infections by regional state over the three time periods, Finland, $1995-2014$

\begin{tabular}{lllllll}
\hline \hline \multirow{2}{*}{ Time period* } & Southern & Southwestern & Western \& & Eastern & Northern & \\
& Finland & Finland & Inland Finland & Finland & Finland & Lapland \\
\hline First & Ref. & Ref. & Ref. & Ref. & Ref. & Ref. \\
Second & $1 \cdot 6(1 \cdot 5-1 \cdot 8)$ & $3 \cdot 8(3 \cdot 2-4 \cdot 5)$ & $1 \cdot 9(1 \cdot 8-2 \cdot 1)$ & $1 \cdot 6(1 \cdot 5-1 \cdot 7)$ & $1 \cdot 4(1 \cdot 3-1 \cdot 5)$ & $1 \cdot 7(1 \cdot 5-1 \cdot 9)$ \\
Third & $2 \cdot 0(1 \cdot 9-2 \cdot 1)$ & $3 \cdot 3(2 \cdot 8-4 \cdot 0)$ & $1 \cdot 8(1 \cdot 7-1-9)$ & $1 \cdot 7(1 \cdot 6-1 \cdot 8)$ & $1 \cdot 1(1 \cdot 0-1 \cdot 2)$ & $0 \cdot 8(0 \cdot 7-0 \cdot 9)$ \\
\hline \hline
\end{tabular}

Values given are incidence rate ratio $(95 \%$ credible interval).

* First period (1 April 1995 to 31 March 2001); Second period (1 April 2001 to 31 March 2007); Third period (1 April 2007 to 31 March 2014).

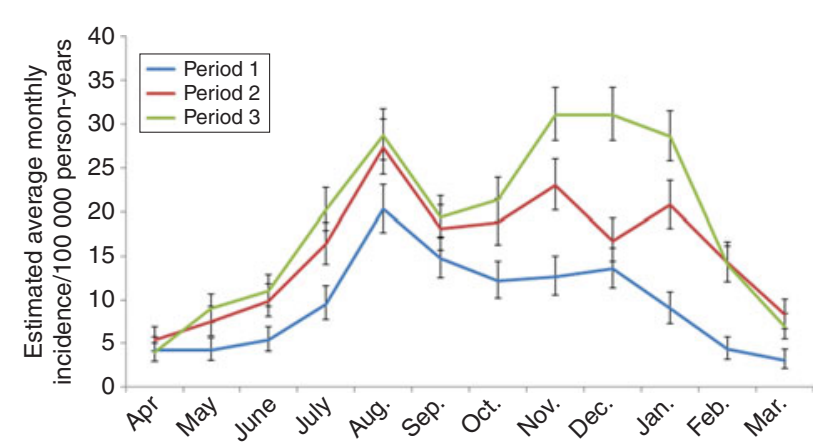

Fig. 2. Average incidence of Puumala hantavirus infection by month in the three time periods, Southern Finland, 1 April 1995-31 March 2014. The vertical bars represent $95 \%$ credible intervals.

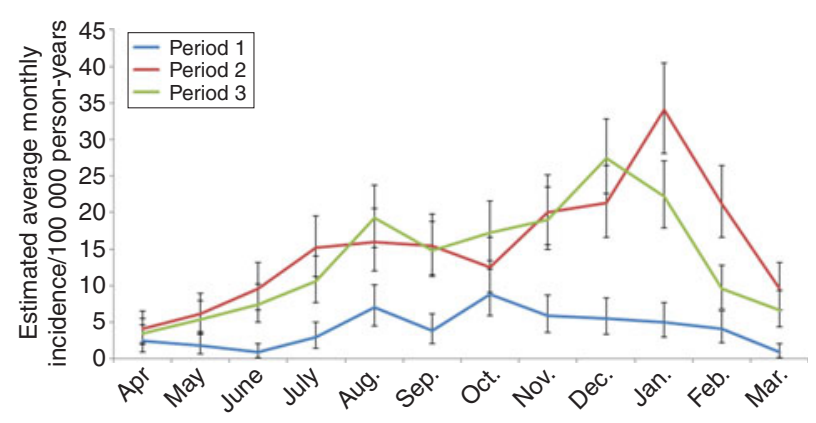

Fig. 3. Average incidence of Puumala hantavirus infection by month in the three time periods, Southwestern Finland, 1 April 1995-31 March 2014. The vertical bars represent $95 \%$ credible intervals.

Marked regional differences were observed in the long-term cycles and seasonality as the cycle lengths increased from south towards north and the pattern of seasonality changed over time in certain areas.

We used 'regional state' as the spatial unit in this study. These administrative regions, unlike the hospital district level often used in epidemiological studies in Finland [7, 9], correspond better although not perfectly with the northern, middle and southern boreal vegetation zones in which the vole populations follow similar dynamics [14]. We divided the study period into three 6-year (first and second periods) or 7 -year (third period) periods, with prior assumptions of cycle length, in order to better allow analyses for temporal changes. Our results on changes in incidence or seasonality are not expected to change significantly if other time division is used given the data on continuous trend. We acknowledge that the true incidence of PUUV infection is higher as only laboratoryconfirmed cases seeking healthcare were included in the numerator in our register-based study.

The increase was highest in Southwestern Finland where incidence had been lowest in the country [7]. The bank-vole cycles may have been weaker during the first period and thus, fewer human PUUV infections were reported. Lack of awareness of PUUV infection and/or diagnostic testing by physicians in the region may also contribute to possible underreporting. Interestingly, the amplitude of vole cycles has shown an increasing trend in this Southwestern area, at least until 2011 [14], which may explain the increased incidence of human PUUV infections. Overall, the increased incidence of PUUV infections in sub-Lapland Finland from the beginning towards the end of the study period closely mirrors the observed increase in amplitude and extent of spatial synchrony in bank-vole population cycles across Finland [3].

The long-term cycle lengths of PUUV infections differed between the south and the north shifting from 3 to 4 years, respectively. These cycle lengths corroborate well with the spatio-temporal population dynamics of bank voles, which are to a large degree influenced by predation and climatic conditions $[1,3,14]$. The pattern in Northern Finland, with a clear 3-year cycle and low but discernible support 


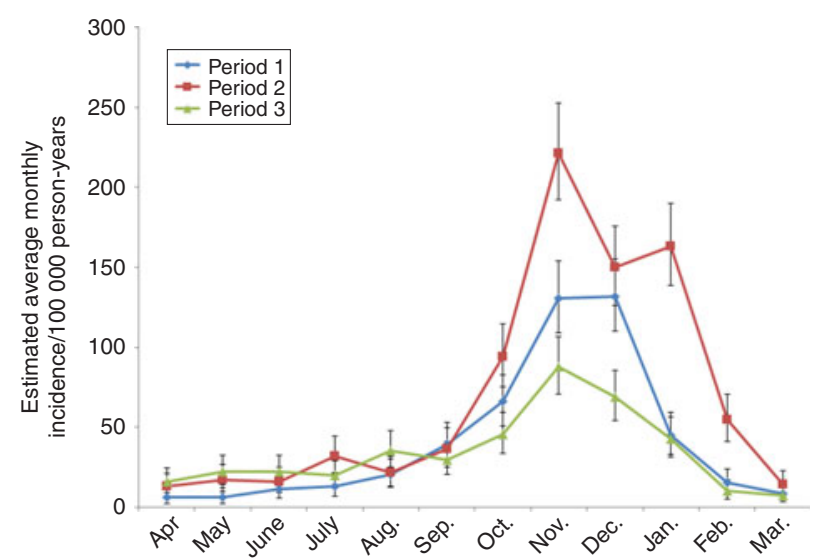

Fig. 4. Average incidence of Puumala hantavirus infection by month in the three time periods, Lapland, 1 April 1995-31 March 2014. The vertical bars represent 95\% credible intervals

for 3.5-year cycle probably reflects the transitional position of this area between the northern and southern vegetation zones.

The regional states exhibited differing HFRS seasonality. In urban areas, particularly in Southern Finland, a clear peak in incidence occurred in August as residents from cities are exposed to the virus during their summer vacations around July in more rural areas (visiting summer cottages), as demonstrated in previous studies [7, 8]. In general and in the other regions, incidence peaked in November or December when rodents looking for shelter due to the approaching winter normally enter human dwellings. It has been demonstrated previously that the increase in incidence follows vole abundance with a lag of a few months [1] although this may vary regionally. Besides the changes in bank-vole populations, aspects of human behaviour, e.g. frequency of human contact with voles and whether protective measures are used, e.g. during handling of firewood or making house repairs, both known risk factors for PUUV infection [15-17], also play a role in the epidemiology. Furthermore, smoking, a wellestablished risk factor for PUUV infection, may play a role in increasing the likelihood of exposure to rodent excreta $[15,18]$. There may be regional differences in the use of protective measures, frequency of visits and activities undertaken in summer cottages.

Temporal changes in seasonality were evident in many regions. In Southern Finland, seasonality was characterized by a clear summer peak in August in the first time period but over time, the winter peak became more apparent (with high incidence until
January) and seasonality in the third period differed from the first. In Southwestern Finland, the seasonality pattern changed from the rather weak seasonal first period towards two rather distinct, albeit relatively weak peaks in the second and third periods. In Lapland, in addition to the significant decrease in incidence in the third period, incidence declined faster from the peak than during the first two periods.

These observed regional changes and differences in human incidence of PUUV infections are probably linked to a complex interaction of temporal and geographical variations in bank-vole population dynamics and climate. A recent study showed that increasing growing-season temperatures have weakened the cyclicity of voles (not specifically bank voles) in cold regions but strengthened cycles in warmer regions of Finland [14]. How these findings translate to temporal and geographical changes in the incidence of human PUUV infections observed in our study warrants further modelling. The model we developed could be utilized for in-depth analyses including bank-vole populations and climatic factors with time lags in order to better understand regional differences in the epidemiology of PUUV infections. Furthermore, regional differences in human behaviour patterns should be assessed with knowledge, attitudes and practice surveys in order to effectively guide intervention measures.

\section{SUPPLEMENTARY MATERIAL}

For supplementary material accompanying this paper visit http://dx.doi.org/10.1017/S0950268816000765.

\section{ACKNOWLEDGEMENTS}

We thank Dr Otso Huitu for critically reviewing the manuscript.

\section{DECLARATION OF INTEREST}

None.

\section{REFERENCES}

1. Vaheri A, et al. Hantavirus infections in Europe and their impact on public health. Reviews in Medical Virology 2013; 23: 35-49.

2. Hardestam $\mathbf{J}$, et al. Puumala hantavirus excretion kinetics in bank voles (Myodes glareolus). Emerging Infectious Diseases 2008; 14: 1209-1215.

3. Olsson GE, Leirs H, Henttonen H. Hantaviruses and their hosts in Europe: reservoirs here and there, but 
not everywhere? Vector Borne and Zoonotic Diseases (Larchmont, NY) 2010; 10: 549-561.

4. Faber MS, et al. Steep rise in notified hantavirus infections in Germany, April 2010. Eurosurveillance 2010; 15: 19574.

5. Ahlm C, et al. Prevalence of serum $\operatorname{IgG}$ antibodies to Puumala virus (haemorrhagic fever with renal syndrome) in northern Sweden. Epidemiology and Infection 1994; 113: 129-136.

6. Zoller L, et al. Seroprevalence of hantavirus antibodies in Germany as determined by a new recombinant enzyme immunoassay. European Journal of Clinical Microbiology \& Infectious Diseases 1995; 14: 305-313.

7. Makary P, et al. Disease burden of Puumala virus infections, 1995-2008. Epidemiology and Infection 2010; 138: 1484-1492.

8. Clement J, et al. Beechnuts and outbreaks of nephropathia epidemica (NE): of mast, mice and men. Nephrology, Dialysis, Transplantation 2010; 25: 1740-1746.

9. Brummer-Korvenkontio M, et al. Epidemiological study of nephropathia epidemica in Finland 1989-96. Scandinavian Journal of Infectious Diseases 1999; 31: 427-435.

10. Olsson GE, et al. Predicting high risk for human hantavirus infections, Sweden. Emerging Infectious Diseases 2009; 15: 104-106.
11. Kallio ER, et al. Cyclic hantavirus epidemics in humans-predicted by rodent host dynamics. Epidemics 2009; 1: 101-107.

12. Congdon P. Bayesian Models for Categorical Data. Chichester: Wiley 2006.

13. Ntzoufras I. Gibbs variable selection using BUGS. Journal of Statistical Software 2002; 7.

14. Korpela K, et al. Nonlinear effects of climate on boreal rodent dynamics: mild winters do not negate highamplitude cycles. Global Change Biology 2013; 19: 697-710.

15. Vapalahti K, et al. Case-control study on Puumala virus infection: smoking is a risk factor. Epidemiology and Infection 2010; 138: 576-584.

16. Gherasim A, et al. Risk factors and potential preventive measures for nephropatia epidemica in Sweden 20112012: a case-control study. Infection Ecology \& Epidemiology 2015; 5: 27698.

17. Van Loock F, et al. A case-control study after a hantavirus infection outbreak in the south of Belgium: who is at risk? Clinical Infectious Diseases 1999; 28: 834-839.

18. Clement JP, et al. Smoking and other risk factors for hantavirus infections: the whole story. Epidemiology and Infection 2011; 139: 1284-1285; author reply 1285-1286. 